\section{A) Check for updates}

Cite this: Org. Biomol. Chem., 2017, 15, 6943

\title{
Transfer hydrogenation of ortho- hydroxybenzophenone ketimines catalysed by BINOL-derived phosphoric acid occurs by a 14-membered bifunctional transition structure $\uparrow$
}

\begin{abstract}
Jolene P. Reid (D) and Jonathan M. Goodman (D) *
Chiral BINOL-derived phosphoric acids catalyse the transfer hydrogenation of ketimines using Hantszch esters. In many cases the nitrogen on the imine binds to the catalyst through the catalyst hydroxyl group and the nucleophile forms a second hydrogen bond to the phosphoryl oxygen. DFT and ONIOM calculations show that the introduction of an ortho-hydroxyaryl group on the carbon atom of the ketimine leads the reaction to proceed through a 14-membered bifunctional mechanism. The transition states of these reactions involve both hydrogen bonding from the hydroxyl group on the imine and the nucleophile's proton to the phosphate catalyst. This mechanistic pathway is lower in energy than the conventional route, consistent with the experimentally observed increased rates of reaction relative to imines that are not derived from ortho-hydroxybenzophenone. To complement the high-level calculations, an accessible qualitative model has been developed that predicts the correct sense of stereoinduction for all examples.
\end{abstract}

Received 2nd June 2017

Accepted 28th July 2017

DOI: $10.1039 / c 7 o b 01345 k$

rsc.li/obc

\section{Introduction}

Chiral phosphoric acids are an important class of Brønsted acids, catalysing a wide range of transformations. ${ }^{1-3}$ The addition of nucleophiles to imines is the largest substrate class for these catalysts. Phosphoric acids catalyse these reactions through a common bifunctional mechanism in which the nitrogen on the imine binds to the phosphoric acid through the Brønsted acidic site while the nucleophile binds through its proton to the $\mathrm{P}=\mathrm{O}$. Therefore, the catalyst simultaneously activates both the nucleophile and the electrophile. This mechanism is known to operate for a wide variety of reactions involving imines. ${ }^{4}$

In 2010 Wang et al. reported that the chiral phosphoric acid catalysed transfer hydrogenation of ketimines facilitated by Hantszch esters could be extended to ortho-hydroxybenzophenone-derived substrates (Fig. 1). ${ }^{5}$ Wang suggested a 10 membered transition state resembling Akiyama's model, ${ }^{6,7}$ with activation of only the imine through protonation and a second hydrogen bonding interaction from the ortho-hydroxylaryl

Centre for Molecular Informatics, Department of Chemistry, University of Cambridge, Lensfield Road, Cambridge CB2 1EW, UK. E-mail:jmg11@cam.ac.uk

$\dagger$ Electronic supplementary information (ESI) available: Complete list of authors in the Gaussian09 reference, Cartesian coordinates, energies, values of imaginary frequencies of the transition state structures and energies of all conformations tested. See DOI: 10.1039/c7ob01345k

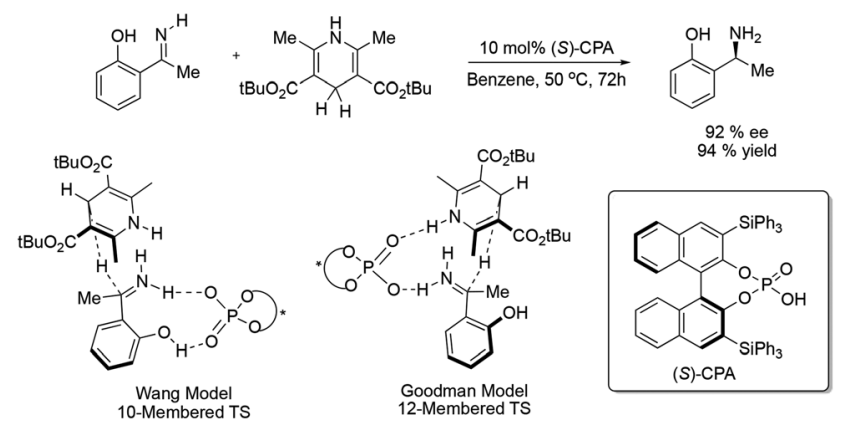

Fig. 1 Asymmetric transfer hydrogenation of ortho-hydroxybenzophenone derived imines.

moiety to the $\mathrm{P}=\mathrm{O}$ of the catalyst. The nucleophile would then be delivered to the most accessible face. Such a model in which only one of the reactants is activated has been known to be higher in energy compared to those involving bifunctional activation. ${ }^{8}$ Despite this, NMR evidence suggested the phenolic proton was bound to the catalyst. ${ }^{5,9}$ The group also noted that removing the hydroxyl group at the ortho position greatly slowed the reaction, supporting the importance of a productive binding site to the catalyst via a hydrogen bond to this position. ${ }^{10}$ The uncertainty associated with the mechanism for this important class of substrates makes prediction of the sense of enantioinduction very difficult for novel reactants. In this 
report we disclose a bifunctional model based on DFT and ONIOM calculations, which suggest that the reaction involves two hydrogen-bonding interactions, one from the orthohydroxyl aryl group on the imine and the second, from the nucleophile's proton to the phosphate catalyst. These interactions lead to a lower energy transition state than those proposed before.

\section{Computational methods}

The preferred reaction pathway was first investigated using buta-1,3-diene-1,4-diol-phosphoric acid as a model for the catalyst, before studying the full molecular system. Quantum mechanical calculations were performed using the Gaussian 09 (revision D.01). ${ }^{11}$ The B3LYP density functional, ${ }^{12,13}$ and split-valence polarized 6-31G** basis set, ${ }^{14,15}$ were used for all geometry optimizations. Single-point energies were taken using the M06-2X density functional, ${ }^{16}$ and $6-31 \mathrm{G}^{* *}$ basis set. ${ }^{14,15}$ This energy was used to correct the gas-phase energy obtained from the B3LYP calculations. For the QM/MM hybrid calculations on the full catalyst, transition states were located first, by a conformational search in MacroModel (version 9.9) ${ }^{17}$ using the OPLS-2005 force field. ${ }^{18-20}$ Selected conformers below $10 \mathrm{~kJ} \mathrm{~mol}^{-1}$ of the minimum were optimized using the ONIOM method implemented in Gaussian 09 (revision D.01). ${ }^{11}$ The B3LYP density functional, ${ }^{12,13}$ and split-valence polarized $6-31 G^{* *}$ basis set, ${ }^{14,15}$ were used for the high-layer, and the force field UFF, ${ }^{21}$ was used for the low-layer. The reactants and the phosphoric acid moiety of the catalyst were included in the high-layer, and the remaining regions of the catalyst were treated as the low-layer. This method has previously been shown to give excellent results when used to describe reactions catalyzed by chiral phosphoric acids. ${ }^{8,22-26}$ The position of the partition within the catalyst was chosen as the phosphoric acid binds directly to the reagents, whereas the remaining catalyst acts as steric bulk and can be adequately described by molecular mechanics. We use the Kekulé bonding structure for all catalysts ensuring that the connectivity in the catalyst backbone is consistent between the structures allowing for accurate energy and geometry comparisons. We have re-optimized the lowest energy TS structures for reaction 1, using the delocalized bonding arrangement and have concluded that it does not affect the relative energies to an appreciable extent. All calculations were performed with the $(S)$-catalyst for model consistency. Single point energy calculations were performed on the resulting structures using M06$2 \mathrm{X}$ density functional, ${ }^{16}$ and the $6-31 \mathrm{G}^{* *}$ basis set, ${ }^{14,15}$ using non-default convergence criteria (fine grid density, ultrafine accuracy level) as implemented in the Jaguar program (version 7.9). ${ }^{27}$ This energy was used to correct the gas-phase energy derived from the ONIOM calculations. Free energies in solution were derived from structures optimized in the gas phase at the ONIOM (B3LYP/6-31G**: UFF), level of theory by means of a single point calculation using M06-2X/6-31G $\mathrm{G}^{* *}$ with the polarizable continuum model (PCM) as implemented in the
Jaguar program (version 7.9), using benzene (probe radius = $2.60 \AA$ ) as the solvent. ${ }^{28}$ These values were used to correct the Gibbs free energy derived from the ONIOM calculations.

\section{Results and discussion}

\section{Mode of activation}

Investigations of the reaction using the simplified phosphoric acid model, buta-1,3-diene-1,4-diol-phosphoric acid considered three different pathways summarised in Fig. 2. To reduce the computational time slightly simpler molecules were used, the di-tert-butyl groups of the Hantzsch ester was replaced by a dimethyl. The transition state in which the catalyst establishes two interactions with the imine (Wang's model) leads to Mechanism A. The study revealed five unique transition states corresponding to this mechanism. Only the lowest energy TS are shown in Fig. 2. The catalyst can establish two points of contact to the nitrogen on the imine and the proton on the Hantszch ester leading to Mechanism B. A total of twelve transition states were located, five of these corresponded to $E$ imine configuration, the rest, $Z$. These transition states where calculated to be disfavoured relative to Mechanism $C$ in which the phosphate catalyst binds to Hantszch ester and the ortho-hydroxyl aryl group. Assuming the reaction is under Curtin-Hammett control, the reaction will proceed via the lowest energy TS. $\mathbf{T S}^{29}$ was found to be the lowest energy and tightest transition state with the shortest hydrogen bonds from imine to catalyst $(1.53 \AA)$. This suggests the binding mode change between the imine and the catalyst is due to increased strength of the hydrogen bonding in Mechanism $C$ relative to Mechanism B. Mechanism $C$ is $7.9 \mathrm{kcal} \mathrm{mol}^{-1}$ lower in energy than the conventional bifunctional Mechanism B. Comparing the $\Delta G^{\ddagger}$ values reveals that the rate of reaction for these substrates would be expected to be faster, the $\Delta G^{\ddagger}$ value for TS3 was calculated to be $3.0 \mathrm{kcal} \mathrm{mol}^{-1}$ which is significantly lower than that obtained for TS2, $10.8 \mathrm{kcal} \mathrm{mol}^{-1}$. These calculations are in excellent agreement with the experimental observations as imines that did not have the ortho-hydroxyl group to

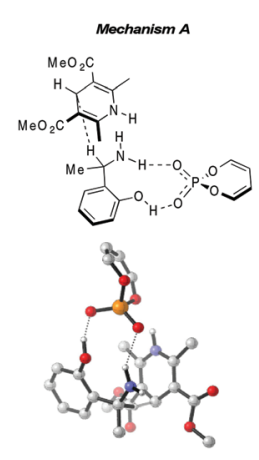

TS1: $\Delta \Delta \mathrm{G}^{\dagger}=+10.4 \mathrm{kcal} \mathrm{mol}^{-1}$

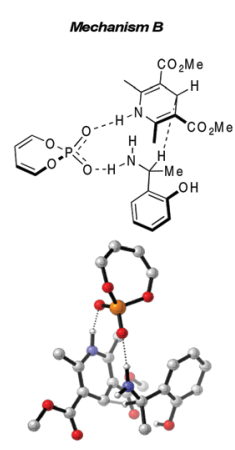

TS2: $\Delta \mathrm{G}^{\ddagger}=+7.9 \mathrm{kcal}^{\mathrm{mol}}{ }^{-1}$

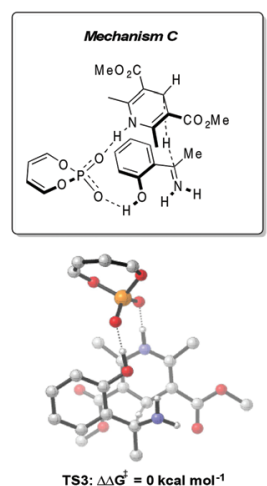

TS3: $\Delta \Delta \mathrm{G}^{\ddagger}=0 \mathrm{kcal} \mathrm{mol}^{-1}$
Fig. 2 Mechanistic proposals and the calculated free energies with the model catalyst. Geometries B3LYP/6-31G**, single-point energy M06$2 X / 6-31 G * *$. 
proceed through this lower energy pathway showed no reaction under the same conditions. ${ }^{10}$ The imine can be activated through a proton transfer from the phosphoric acid or the $\mathrm{OH}$

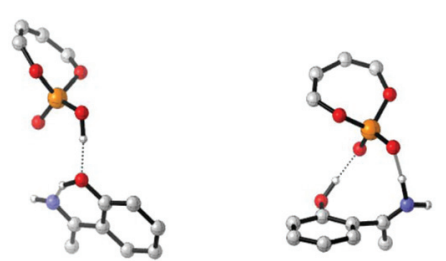

Fig. 3 TSs for the protonation of imine catalysed by a model phosphoric acid. All energies in $\mathrm{kcal} \mathrm{mol}^{-1}$. Geometries B3LYP/6-31G**, singlepoint energy M06-2X/6-31G**. group on the aryl ring. We have characterized both pathways and our calculations indicate that the intramolecular proton transfer is most feasible (Fig. 3).

\section{Model for stereoselectivity}

Transition states for the full catalyst system were located using ONIOM (B3LYP/6-31G**: UFF), single-point energies M06-2X/ $6-31 G^{* *}$ were then calculated for the low energy reaction pathways (see ESI $\dagger$ for further details). Solvation free energy corrections were computed by means of the PCM model. We have previously identified this as an excellent computational method for studying chiral phosphoric acid catalysed transfer hydrogenation reactions. ${ }^{8,26}$ There are ten reasonable transition states for these reactions, resulting from three mecha-

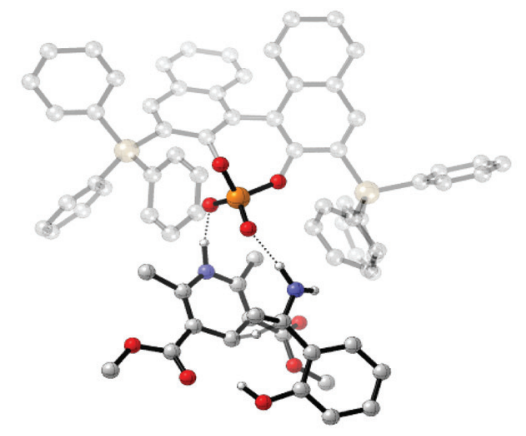

TS4-Z: $\Delta \Delta \mathrm{G}^{\ddagger}=+12.8 \mathrm{kcal} \mathrm{mol}^{-1}, \Delta \Delta \mathrm{G}_{\mathrm{sol}}^{\ddagger}=+12.5 \mathrm{kcal} \mathrm{mol}^{-1}$

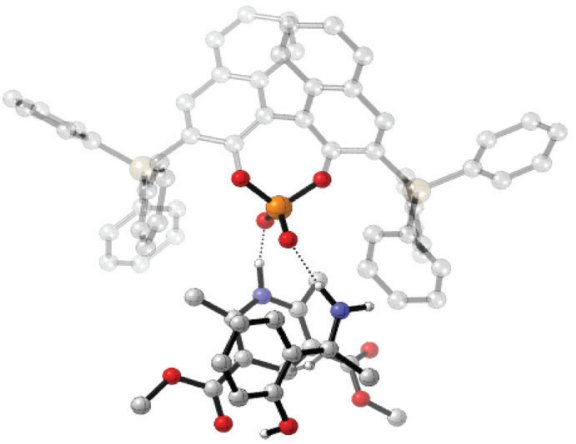

TS4-E: $\Delta \Delta \mathrm{G}^{\ddagger}=+9.5 \mathrm{kcal} \mathrm{mol}^{-1}, \Delta \Delta \mathrm{G}_{\mathrm{sol}}{ }^{\ddagger}=+9.9 \mathrm{kcal} \mathrm{mol}^{-1}$

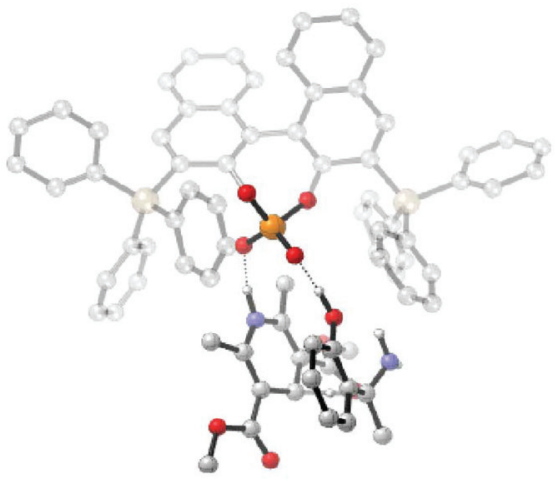

TS6-E: $\Delta \Delta \mathrm{G}^{\ddagger}=0 \mathrm{kcal} \mathrm{mol}^{-1}, \Delta \Delta \mathrm{G}_{\mathrm{sol}}{ }^{\ddagger}=0 \mathrm{kcal} \mathrm{mol}^{-1}$

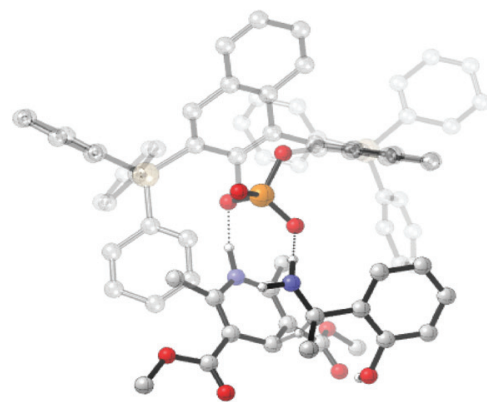

TS5-E: $\Delta \Delta G^{\ddagger}=+12.9 \mathrm{kcal} \mathrm{mol}^{-1}, \Delta \Delta \mathrm{G}_{\text {sol }}^{\ddagger}=+13.1 \mathrm{kcal} \mathrm{mol}^{-1}$

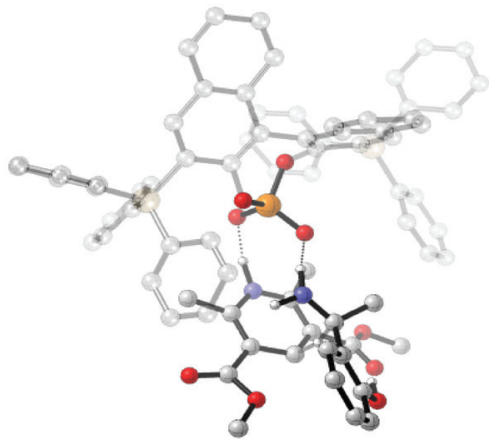

TS5-Z: $\Delta \Delta \mathrm{G}^{\ddagger}=+11.3 \mathrm{kcal} \mathrm{mol}^{-1}, \Delta \Delta \mathrm{G}_{\mathrm{sol}}{ }^{\ddagger}=+11.5 \mathrm{kcal} \mathrm{mol}^{-1}$

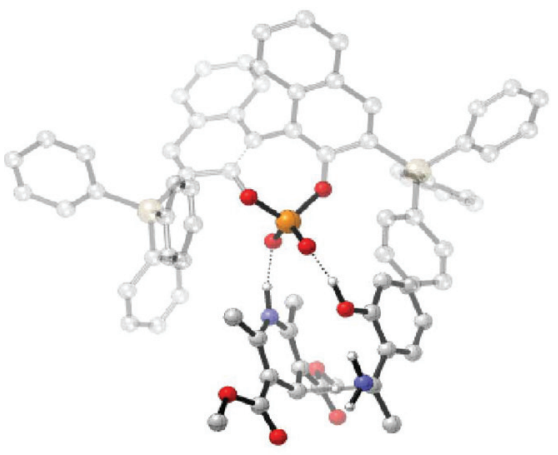

TS7-E: $\Delta \Delta G^{\ddagger}=+1.9 \mathrm{kcal} \mathrm{mol}^{-1}, \Delta \Delta \mathrm{G}_{\mathrm{sol}}{ }^{\ddagger}=+1.4 \mathrm{kcal} \mathrm{mol}^{-1}$

Fig. 4 Competing TSs for the $\mathrm{SiPh}_{3}$ catalysed transfer hydrogenation reaction. ONIOM (B3LYP/6-31G**: UFF), single-point energy M06-2X/6$31 \mathrm{G}^{* *}$. Grayed-out regions were treated with UFF, and the full-colour regions were treated B3LYP/6-31G**. Structures generated using CYLview. ${ }^{30}$ 
nisms (Fig. 2) and a number of different arrangements of the imine. Our model studies allowed us to reduce this number to eight, as the energy differences between the dual (Mechanism $A$ ) and bifunctional modes (Mechanisms $B$ and $C$ ) of activation are so large that not even the catalysts steric interactions could change this preference. The lowest energy conformations of the transition states are shown in Fig. 4, energies of additional conformations have been provided in the ESI. $\dagger$ For each of the bifunctional mechanisms the $\mathrm{N}$-substituent of the imine can be directed towards the front of the 3,3' substituents on the binaphthol ring leading to a Type II reaction pathway or away, Type $I$. Additionally the imine can exist as either the $E$ or $Z$ isomers for the $\mathrm{NH}$ binding mode. The $\mathrm{OH}$ binding mode is restricted to proceed via the $E$ configuration, geometry optimizations of $Z$ TSs showed that these structures converged into the dual mode of activation (Mechanism A) and were disfavoured by $18.8 \mathrm{kcal} \mathrm{mol}^{-1}$ or more relative to TS6- $\boldsymbol{E}$. Therefore TS's involving the $Z$ configuration and this binding mode was not investigated further. Thorough conformational searching of the possible catalyst-substrate conformations located a total of 72 transition states (see ESI $\dagger$ ). The calculations suggest that the reaction proceeds via an OH-Type II E

Table 1 Interatomic distances for the lowest energy ONIOM TSs

\begin{tabular}{lllll}
\hline \multicolumn{5}{c}{ Interatomic distance $(\AA)$} \\
\cline { 2 - 5 } & $\begin{array}{l}\text { C-H } \\
\text { (breaking) }\end{array}$ & $\begin{array}{l}\text { C-H } \\
\text { (forming) }\end{array}$ & $\begin{array}{l}\text { NH/OH-O } \\
\text { (imine) }\end{array}$ & $\begin{array}{l}\text { NH-O } \\
\text { (Hantzsch ester) }\end{array}$ \\
\hline TS4- $Z$ & 1.39 & 1.34 & 1.76 & 1.50 \\
TS5- $\boldsymbol{E}$ & 1.42 & 1.32 & 1.70 & 1.64 \\
TS4- $\boldsymbol{E}$ & 1.40 & 1.32 & 1.81 & 1.50 \\
TS5- $Z$ & 1.37 & 1.35 & 1.76 & 1.59 \\
TS6- $\boldsymbol{E}$ & $\mathbf{1 . 3 6}$ & $\mathbf{1 . 3 2}$ & $\mathbf{1 . 4 8}$ & $\mathbf{1 . 5 6}$ \\
TS7- $\boldsymbol{E}$ & 1.36 & 1.33 & 1.50 & 1.61
\end{tabular}

reaction mechanism, TS6- $\boldsymbol{E}$, this is favoured relative to the Type I E, TS7-E, by $1.9 \mathrm{kcal} \mathrm{mol}^{-1}$. This is in good agreement with the experimentally observed sense of enantioselectivity. The ortho-hydroxy aryl group of the imine prefers to be pointed away from the catalyst 3,3' substituents and occupies the free space at the front left hand side of the catalyst. The imine adopts a tilted position, minimising the steric interactions of the $N$-substituent and the $3,3^{\prime}$ group. In the Type I E TS the aromatic group is orientated towards the back phenyl ring of the $\mathrm{SiPh}_{3} 3,3^{\prime}$ substituent. This leads to a large energetic penalty and increases the energy of the Type I E TS relative to the Type II E. The NH Type II E TS also avoids the unfavourable steric interactions between the ortho-hydoxy group and the 3,3' substituent. However, despite the structural similarities, the $\mathrm{NH}$ binding mode was strongly disfavoured confirming the same trends as the model catalyst. The most favourable TS, TS-6 $\boldsymbol{E}$ was the tightest of all the TSs with the shortest hydrogen bonds. The strength of the hydrogen bond between TS6-E and TS4- $\boldsymbol{E}$ is compromised; the angle of the hydrogen bond deviates modestly away from linearity $176^{\circ}$ to $171^{\circ}$ and lengthens from $1.48 \AA$ to $1.81 \AA$. Therefore, the reasons for the large energetic preference of OH-Type II E TS originates from both steric and electronic factors. Relative populations of transition states were predicted at the temperature they were performed from the corrected Gibbs free energy differences and based on Boltzmann distribution analysis. The computed enantiomeric excess (ee) was $90 \%$, which is very close to the experimental value $(92 \%)$ (Table 1$)$.

We have summarised the calculations into a qualitative model (Fig. 5, bottom, the $\mathrm{OOH}$ model for ortho-OH substrates) to explain and predict the stereochemical outcome in the chiral phosphoric acid catalysed transfer hydrogenations of ortho-hydroxy ketimines. The model is built on the $\mathrm{OH}$ binding mode in which the aromatic substituent is orientated towards the empty pocket of the catalyst. Increasing the sterics

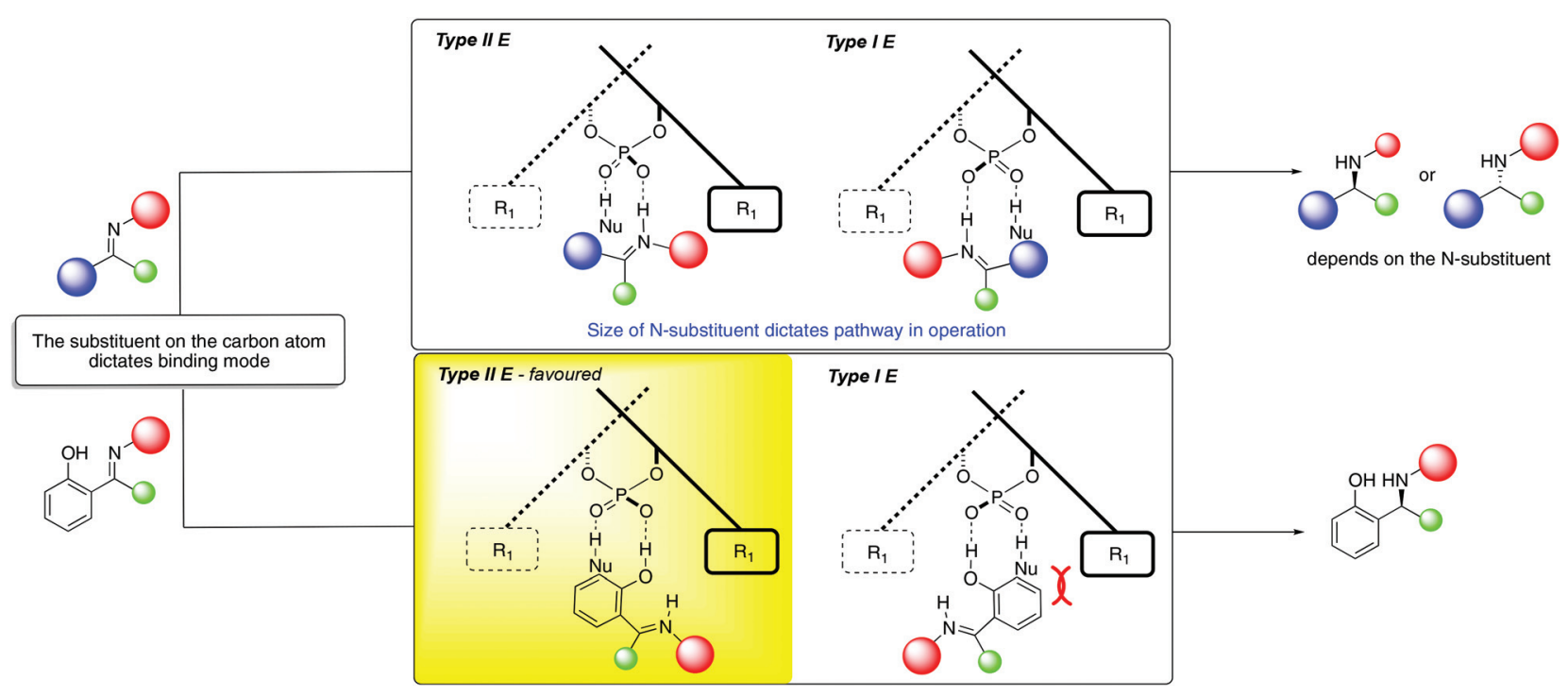

Fig. 5 Conventional (top) and $\mathrm{OOH}$ (ortho- $\mathrm{OH}$, bottom) qualitative models for predicting stereoselectivity for nucleophilic additions to imines. 
remote from the phosphoric acid maximises the steric repulsions between the ortho-hydroxy aryl group and the $3,3^{\prime}$ substituents in the Type $I E$ TS leading to high levels of enantioselectivity.

The qualitative $\mathrm{OOH}$ model predicts that the introduction of a larger substituent on the nitrogen indicated in red, Fig. 5, would result in a minimal increase in steric repulsions between this substituent and the 3,3' group. However, the conventional bifunctional model ${ }^{4}$ (Fig. 5, top) predicts major steric repulsions and a reaction pathway change from Type II to Type $I$ as a result. To test this, transition states for a second literature reaction, ${ }^{10}$ reported by Wang et al. in which the $\mathrm{H}$ on the nitrogen was replaced by a aromatic moiety were located using ONIOM (B3LYP/6-31G**: UFF), the results are summarized in the ESI. $\dagger$ To simplify our calculations, the aromatic moiety was truncated to a phenyl group; calculations on similar systems have reported this to have a minimal effect on the reaction outcome but would rapidly enhance computational tractability in terms of conformational flexibility. ${ }^{8,26}$ According to our calculations OOH-Type II $E$ is favoured over OOH-Type I $E$ by $2.0 \mathrm{kcal} \mathrm{mol}^{-1}$ a value very similar to that calculated for the reduction of $\mathrm{NH}$ ketimines and in good agreement experiment, $89 \%$ ee. However, for the $\mathrm{NH}$ binding mode the lowest energy pathway is now Type $I Z$ due to the increased steric interactions between the $N$-aryl substituent and the $3,3^{\prime}$ groups in the Type II $E$ TS. The standard bifunctional mechanism would predict a mechanism change where the $\mathrm{OOH}$ model does not. The $\mathrm{OOH}$ model is consistent with the experimental data.

\section{Conclusions}

DFT and ONIOM calculations suggest that the phosphoric acid catalysed transfer hydrogenation of imines derived from orthohydroxybenzophenone involves a bifunctional mechanism in which there are two hydrogen-bonding interactions from reagents to catalyst, one from the ortho-hydroxyl group and a second from the nucleophile's proton. This mode of activation is lower in energy than those originally proposed. The hydroxyl group is a key element in dictating reactivity and enantioselectivity. This is an under-appreciated mode of activation achieving faster rates of reaction than the conventional bifunctional mode and these investigations should promote future developments in the field. Our calculations have been summarized into a simple qualitative model (Fig. 5) that accurately reproduces the experimentally observed sense of enantioinduction for all examples. This model highlights the important steric interactions between substrate and catalyst necessary for efficient stereoinduction.

\section{Acknowledgements}

We thank the EPSRC for funding.

\section{References}

1 T. Akiyama, Chem. Rev., 2007, 107, 5744.

2 M. Terada, Synthesis, 2010, 1929.

3 D. Parmar, E. Sugiono, S. Raja and M. Rueping, Chem. Rev., 2014, 114, 9047.

4 J. P. Reid, L. Simón and J. M. Goodman, Acc. Chem. Res., 2016, 49, 1029.

5 T. B. Nguyen, H. Bousserouel, Q. Wang and F. Gueŕitte, Org. Lett., 2010, 12, 4705.

6 T. Akiyama, J. Itoh, K. Yokota and K. Fuchibe, Angew. Chem., Int. Ed., 2004, 43, 1566.

7 M. Yamanaka, J. Itoh, K. Fuchibe and T. Akiyama, J. Am. Chem. Soc., 2007, 129, 6756.

8 L. Simón and J. M. Goodman, J. Am. Chem. Soc., 2008, 130, 8741.

9 T. B. Nguyen, Q. Wang and F. Gueŕitte, Chem. - Eur. J., 2011, 17, 9576.

10 T. B. Nguyen, H. Bousserouel, Q. Wang and F. Gueŕitte, Adv. Synth. Catal., 2011, 353, 257.

11 M. J. Frisch, et al., Gaussian 09, Revision D.01, Gaussian, Inc., Wallingford, CT, 2013.

12 A. D. Becke, Phys. Rev. A, 1988, 38, 3098.

13 C. Lee, W. Yang and R. G. Parr, Phys. Rev. B: Condens. Matter, 1988, 37, 785.

14 R. Krishnan, J. S. Binkley, R. Seeger and J. A. Pople, J. Chem. Phys., 1980, 72, 650.

15 P. M. W. Gill, B. G. Johnson, J. A. Pople and M. J. Frisch, Chem. Phys. Lett., 1992, 197, 499.

16 Y. Zhao and D. Truhlar, Theor. Chem. Acc., 2008, 120, 215.

17 MacroModel, version 9.9, Schrodinger, LLC, New York, NY, 2009.

18 G. A. Kaminski, R. A. Friesner, J. Tirado-Rives and E. Jorgensen, J. Phys. Chem. B, 2001, 105, 6474.

19 W. L. Jorgensen, D. S. Maxwell and J. Tirado-Rives, J. Am. Chem. Soc., 1996, 118, 11225.

20 W. L. Jorgensen and J. Tirado-Rives, J. Am. Chem. Soc., 1988, 110, 1657.

21 A. K. Rappe, C. J. Casewit, K. S. Colwell, W. A. Goddard and W. M. Skiff, J. Am. Chem. Soc., 1992, 114, 10024.

22 L. Simón and J. M. Goodman, J. Am. Chem. Soc., 2009, 131, 4070.

23 L. Simón and J. M. Goodman, J. Org. Chem., 2010, 75, 589.

24 M. N. Grayson, S. C. Pellegrinet and J. M. Goodman, J. Am. Chem. Soc., 2012, 134, 2716.

25 L. M. Overvoorde, M. N. Grayson, Y. Luo and J. M. Goodman, J. Org. Chem., 2015, 80, 2634.

26 J. P. Reid and J. M. Goodman, J. Am. Chem. Soc., 2016, 138, 7910.

27 Jaguar, version 7.9, Schrodinger, LLC, New York, NY, 2012.

28 B. Mennucci and J. Tomasi, J. Chem. Phys., 1997, 106, 5151.

29 We have drawn the TS as aromatic but other resonance structures are possible.

30 CYL view, 1.0b, Université de Sherbrooke, Legault, C. Y., 2009. 